\title{
The Use of Osteochondral Allograft Transplantation for Primary Treatment of Cartilage Lesions in the Knee
}

Cartilage

2015, Vol. 6(4) 203-207

(c) The Author(s) 2015

Reprints and permissions:

sagepub.com/journalsPermissions.nav DOI: $10.1177 / 1947603515595072$

cart.sagepub.com

@SAGE

\author{
Dustin T. Briggs', Kamran N. Sadr², Pamela A. Pulido ${ }^{3}$, \\ and William D. Bugbee ${ }^{3,4}$
}

\begin{abstract}
Objective:To assess the outcome of osteochondral allograft (OCA) transplantation as the primary treatment for cartilage injury in patients with no previous surgical treatment. Study Design: Case series. Patients were identified in our outcomes database. Patients undergoing primary OCA transplantation with no prior surgical treatment and a minimum of 2 years follow-up were selected. Pain and function were evaluated preoperatively and postoperatively. Patient satisfaction was assessed. Reoperations following OCA transplantation were captured. Failure was defined as revision OCA or conversion to arthroplasty. Results: Fifty-five patients (6I knees) were included in the analysis. The study consisted of 30 males and 25 females (mean age $=32.9$ years; range $=15.7-67.8$ years). The most common diagnoses for the OCA transplantation were osteochondritis dissecans (44.3\%) and avascular necrosis (31.1\%). Pain and function improved preoperatively to postoperatively on all outcome scales $(P<0.01)$. The majority of patients $(86 \%)$ were "extremely satisfied" or "satisfied." OCA survivorship was $89.5 \%$ at 5 years and $74.7 \%$ at 10 years. At latest follow-up (mean $=7.6$ years; range $=1.9-22.6$ years), OCA remained in situ in 50 knees (82\%). Eighteen knees (29.5\%) had further surgery; I I OCA failures and 7 other surgical procedure(s). Of the failed knees (mean time to failure $=3.5$ years; range $=0.5-13.7$ years), 8 were converted to arthroplasty, 2 had OCA revisions, and I had a patellectomy. Conclusions: OCA transplantation is an acceptable primary treatment method for some chondral and osteochondral defects of the knee. Failure of previous treatment(s) is not a prerequisite for OCA transplantation.
\end{abstract}

\section{Keywords}

osteochondral allograft transplantation, knee, cartilage repair

\section{Introduction}

Injury to hyaline cartilage continues to present a difficult clinical problem. Multiple cartilage repair techniques, including loose-body fixation, debridement, microfracture, osteochondral autologous transplantation (OAT), autologous chondrocyte implantation (ACI), and prosthetic resurfacing, have been suggested as possible treatment modalities for various cartilage disorders. ${ }^{1-4}$ Selection of the appropriate repair technique typically requires consideration of numerous factors. In most treatment algorithms, osteochondral allograft (OCA) transplantation is regarded as a salvage procedure when previous treatments have failed ${ }^{1}$; thus, outcomes of OCA transplantation, when used as a primary treatment option, is not well known. In OCA transplantation, an equivalent-sized fragment of fresh allograft cartilage, with supportive subchondral bone, is transplanted into the cartilage defect. ${ }^{5-15}$ This technique dates back to as early as $1914^{16}$ and restores the defect to an architecturally accurate, mature hyaline cartilage. ${ }^{17}$
Numerous studies have reported the outcome of OCA transplantation; however, most patients in these studies have had previous surgery in an attempt to treat the cartilage disease prior to the OCA transplantation. Nonetheless, the majority of patients experienced improved function and good to excellent overall repair with graft survivorship of $>80 \%$ at 10 years or less and $74 \%$ at 15 years. ${ }^{6,8,13}$ In these studies and others, over $90 \%$ of the patients had undergone previous surgical treatment prior to the allograft procedure.

\footnotetext{
'Department of Orthopaedics, University of New Mexico, Albuquerque, NM, USA

${ }^{2}$ Kaiser Permanente, Freemont, CA, USA

${ }^{3}$ Shiley Center for Orthopaedic Research \& Education at Scripps Clinic, La Jolla, CA, USA

${ }^{4}$ Division of Orthopaedics, Scripps Clinic, La Jolla, CA, USA

Corresponding Author:

William D. Bugbee, Scripps Clinic, 10666 N. Torrey Pines Rd., MSI I6, La Jolla, CA 92037, USA.

Email: Bugbee.william@scrippshealth.org
} 
Table I. Patient Characteristics and OCA Details $(N=55$ Patients; 6I Knees).

\begin{tabular}{lcc}
\hline Variable & Mean (SD) or \% & Range \\
\hline Age (years) by patients & $32.9(16.0)$ & $15-67$ \\
Male (\%) by patients & 54.5 & \\
Diagnosis by knees (\%) & & \\
Osteochondritis dissecans & 44.3 & \\
Avascular necrosis & 31.1 & \\
Osteoarthritis & 8.2 & \\
Traumatic chondral injury & 6.6 & \\
Degenerative chondral lesion & 6.6 & \\
Fracture & 3.2 & \\
Graft location by knees (\%) & & \\
Femoral condyle, medial (MFC) & 47.5 & \\
Femoral condyle, lateral (LFC) & 24.6 & \\
Patella, trochlea & 8.2 & \\
Trochlea & 4.9 & \\
MFC, LFC & 4.9 & \\
Patella & 3.3 & \\
Tibia plateau, lateral (LTP) & 1.6 & \\
Tibia plateau, medial (MTP, MFC) & 1.6 & \\
MFC, Patella & 1.6 & \\
MFC, Patella, Trochlea & 1.6 & \\
Number of grafts by knees (\%) & 57.4 & \\
One & 36.1 & \\
Two & 6.6 & \\
Three & $9.6(6.2)$ & \\
Total graft area (cm ${ }^{2}$ ) & & \\
\hline
\end{tabular}

Patient age ( $>30$ years) and multiple operations have been associated with higher rates of graft failure. ${ }^{13}$ From this literature, it is evident that prior treatments may be a confounding factor in assessing OCA repair outcomes. Thus, the objective of this study was to assess the outcome of OCA transplantation when it was used as the primary treatment for cartilage injury in the knee in patients with no previous surgical treatment.

\section{Methods}

Since 1983, OCA transplantation data have been collected from 877 patients and recorded in an outcomes database under an institutional review board-approved protocol. All patients in the database signed an informed consent prior to any study related procedures. For this study, the database was used to identify patients who underwent OCA transplantation as primary treatment for a chondral or osteochondral defect. Inclusion criteria include patients, of any age, with no prior surgical treatment of an isolated, Grade III or IV chondral or osteochondral defect who were treated with OCA and had a minimum 2-year follow-up. Exclusion criteria included any previous surgery on the affected knee, advanced degenerative arthritis affecting more than one compartment of the knee, uncorrected limb malalignment, or ligamentous instability.

All procedures involved OCA transplantations in the knee, utilizing fresh unmatched tissue. OCA transplantation was selected as the primary treatment option based on disease state, defect size, involvement of bone along with the cartilage lesion, and patient age. Grafts were processed in accordance with standards of the American Association of Tissue Banks either at (1) the regional tissue bank at the local university and stored at $4{ }^{\circ} \mathrm{C}$ in lactated Ringer solution containing $1 \mathrm{~g} / \mathrm{L}$ of cefazolin and $10 \mathrm{~g} / \mathrm{mL}$ of gentamicin for 2 to 6 days $(N=23)$ or $(2)$ at a commercial tissue bank and stored at $4^{\circ} \mathrm{C}$ in proprietary tissue culture medium $(N=38)$. The surgical procedure has been previously described. ${ }^{17}$

Preoperative and postoperative pain and function were obtained using the modified Merle d'Aubigné-Postel (18-point) scale; the subjective International Knee Documentation Committee (IKDC) pain, function, and total scores; the Knee Society function (KS-F) and knee (KS-K) scores; and the Knee injury and Osteoarthritis Outcome Score (KOOS). Patient satisfaction was assessed using a 5-point scale from "extremely satisfied" to "extremely dissatisfied." After the initial OCA transplantation, the number and type of further surgeries on the operative joint were captured. Graft failure was defined as revision of the OCA or conversion to arthroplasty.

\section{Statistical Analysis}

All statistical analyses were performed with SPSS version 13.0 (IBM Corporation, Armonk, NY). Patient characteristics, allograft details (such as diagnosis, graft size, and graft site), and the number and type of further surgery on the operative knee were summarized using means and frequencies. Wilcoxon signed-ranks tests were used to assess change from preoperative to postoperative (at latest follow-up) outcome scores on the modified Merle d'Aubigné-Postel (18-point), IKDC, KS-F, KS-K, and KOOS scales. The Kaplan-Meier method was used to calculate allograft survivorship with graft failure as the endpoint. A $P$ value of 0.05 was used to determine statistical significance.

\section{Results}

Fifty-five patients (61 knees) met all of the inclusion criteria and none of the exclusion criteria and were included in the final analysis (Table 1). The patient population consisted of 30 males $(54.5 \%)$ and 25 females (45.5\%), with a mean age of 32.9 years (range $=15.7-67.8$ years). The most common diagnoses for the OCA transplantation were OCD $(44.3 \%)$ and avascular necrosis (AVN; 31.1\%); other diagnoses occurred at lower frequency and included osteoarthritis $(8.2 \%)$, traumatic chondral injury $(6.6 \%)$, degenerative chondral lesions $(6.6 \%)$, and fracture $(3.2 \%)$. The mean graft size was $9.6 \mathrm{~cm}^{2}$ with a range of $3.2 \mathrm{~cm}^{2}$ to $34.8 \mathrm{~cm}^{2}$, with graft sites most commonly occurring in the medial femoral condyle $(47.5 \%)$ or lateral femoral condyle $(24.6 \%)$. The majority of knees had one OCA $(57.4 \%)$, with the remaining knees having $2(36.1 \%)$ or $3(6.6 \%)$ grafts. Fourteen of 61 
Table 2. Results of Subjective Outcome Measures.

\begin{tabular}{|c|c|c|c|c|}
\hline Measure & $n$ & Preoperative Mean (SD) or \% & Postoperative Mean (SD) or \% & $P$ Value $^{\mathrm{a}}$ \\
\hline Modified Merle d’Aubigné-Postel (I8-point) & 44 & $12.6(1.9)$ & $16.5(1.9)$ & $<0.001$ \\
\hline Excellent & & - & $35.6 \%$ & \\
\hline Good & & $14.3 \%$ & $48.9 \%$ & \\
\hline Fair & & $55.1 \%$ & $13.3 \%$ & \\
\hline Poor & & $30.6 \%$ & $2.2 \%$ & \\
\hline \multicolumn{5}{|l|}{ IKDC } \\
\hline Pain & 35 & $6.2(1.8)$ & $2.2(2.5)$ & $<0.001$ \\
\hline Function & 36 & $2.8(1.4)$ & $7.9(2.1)$ & $<0.001$ \\
\hline Total score & 32 & $36.9(9.7)$ & $80.4(16.8)$ & $<0.001$ \\
\hline \multicolumn{5}{|l|}{ Knee Society } \\
\hline Function & 33 & $66.5(14.9)$ & $89.7(21.4)$ & $<0.001$ \\
\hline Knee score & 12 & $76.7(18.8)$ & $92.3(11.0)$ & 0.006 \\
\hline \multicolumn{5}{|l|}{ KOOS } \\
\hline Symptoms & 13 & $59.2(17.4)$ & $84.9(16.8)$ & $<0.001$ \\
\hline Pain & 13 & $57.9(16.0)$ & $88.2(17.5)$ & $<0.001$ \\
\hline ADL & 13 & $63.7(16.3)$ & $91.9(16.0)$ & $<0.001$ \\
\hline Sport/Recreation & 9 & $38.3(28.6)$ & $81.1(11.1)$ & 0.001 \\
\hline QOL & 13 & $22.2(17.0)$ & $65.5(22.4)$ & $<0.001$ \\
\hline Satisfaction & 44 & & & \\
\hline Extremely satisfied & & - & 77.3 & \\
\hline Satisfied & & - & 9.1 & \\
\hline Somewhat satisfied & & - & 11.4 & \\
\hline Dissatisfied & & - & 2.3 & \\
\hline
\end{tabular}

IKDC = International Knee Documentation Committee; KOOS = Knee injury and Osteoarthritis Outcome Score; $A D L=$ activities of daily living; $\mathrm{QOL}=$ quality of life.

${ }^{a}$ Wilcoxon signed ranks test.

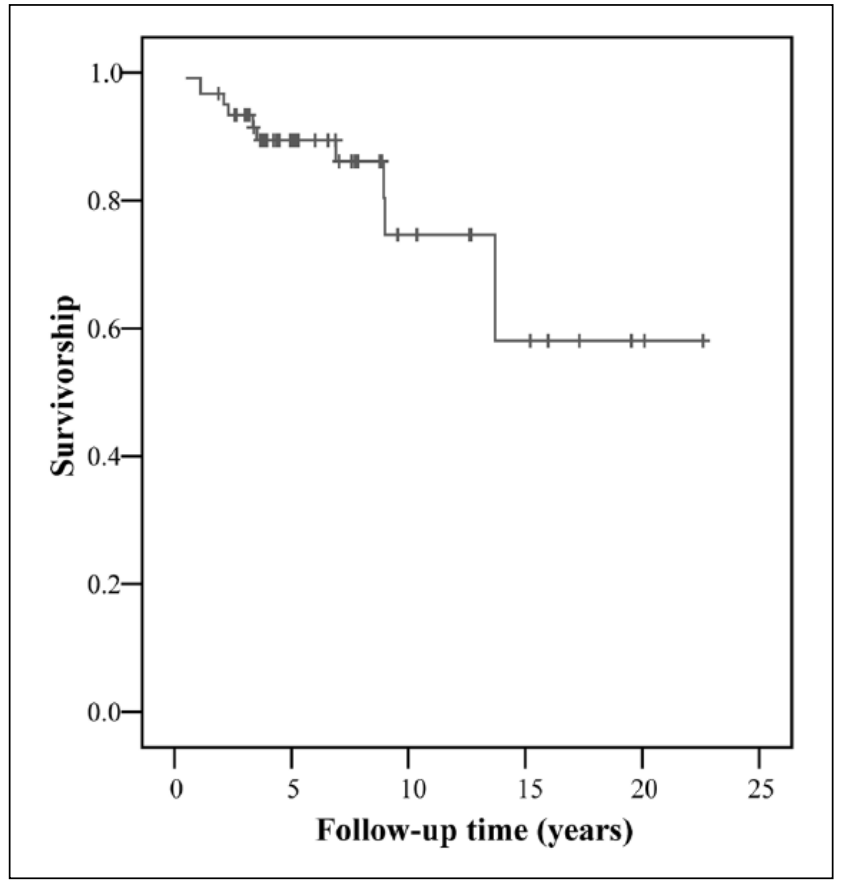

Figure I. Survivorship of OCA transplant as primary treatment was $89.5 \%$ at 5 years and $74.7 \%$ at 10 years.
(23.0\%) knees had an additional procedure performed at the time of OCA including lateral release (5), marrow stimulation (3), high tibial osteotomy (2), menisectomy (2), diagnostic arthroscopy (1), and ACL reconstruction (1).

At latest follow-up, 50 knees $(82.0 \%)$ had the OCA in situ, with a mean follow-up duration for such knees of 7.6 years (range $=1.9-22.6$ years). Pain and function improved from preoperatively to postoperatively on all of the outcome scales including the modified Merle d'Aubigné-Postel (18-point), IKDC, KS-F, KS-K, and KOOS (all, $P<0.01$; Table 2). In terms of patient satisfaction, $86 \%$ of patients reported being "extremely satisfied" or "satisfied."

OCA survivorship was $89.5 \%$ at 5 years and $74.7 \%$ at 10 years (Fig. 1). Following the initial OCA transplantation, 18 knees $(29.5 \%)$ had further surgery. Of these additional surgeries, 11 knees (10 patients) had a surgical procedure to address an OCA failure or progression of arthritis and 7 knees ( 9 surgeries total) had surgical procedure(s) performed in conjunction with a functional graft. Additional surgeries performed on knees in conjunction with a functional graft included arthroscopic debridement and/or loose body removal (6), OAT (1), meniscus repair (1), and lateral release (1). Of the 11 failed knees, 8 knees were converted to arthroplasty, 2 knees had an OCA revision, and 1 knee had a 
Table 3. Details of II OCA Failures in IO Patients.

\begin{tabular}{|c|c|c|c|c|c|c|}
\hline Year of Surgery & $\begin{array}{c}\text { Age at Time of OCA } \\
\text { (years) }\end{array}$ & Number of Grafts & Location & OCA Diagnosis & $\begin{array}{c}\text { Time to Failure } \\
\text { (years) }\end{array}$ & Failure Type \\
\hline 1984 & 64.2 & I & Patella & $\mathrm{DCL}$ & I.I & OCA revision \\
\hline 1993 & 44.0 & 2 & Patella, trochlea & OA & 2.3 & Patellectomy \\
\hline 1998 & 64.2 & 2 & Patella, trochlea & OA & 9.0 & TKA \\
\hline 1999 & 50.9 & 2 & Patella, trochlea & $\mathrm{DCL}$ & 3.5 & TKA \\
\hline 1999 & 55.8 & 2 & Patella, trochlea & OA & 6.9 & TKA \\
\hline \multirow[t]{2}{*}{2000} & 17.3 & 1 & LFC & AVN & 13.7 & $\mathrm{TKA}^{\mathrm{a}}$ \\
\hline & & 1 & LFC & AVN & 13.7 & $\mathrm{TKA}^{\mathrm{a}}$ \\
\hline 2001 & 30.4 & I & Patella & AVN & 0.5 & TKA \\
\hline 2001 & 35.8 & 2 & MFC, LFC & AVN & 8.9 & TKA \\
\hline 2005 & 18.2 & 2 & MFC, LFC & AVN & 3.3 & OCA revision \\
\hline 2005 & 66.0 & 2 & MFC, MTP & OA & 2.1 & TKA \\
\hline
\end{tabular}

$\mathrm{DCL}=$ degenerative chondral lesion; $\mathrm{OA}=$ osteoarthritis; $\mathrm{AVN}=$ avascular necrosis; $\mathrm{OCA}=$ osteochondral allograft; $\mathrm{TKA}=$ total $\mathrm{knee}$ arthroplasty;

LFC = lateral femoral condyle; $M F C=$ medial femoral condyle; $M$ TP = medial tibial plateau.

${ }^{a}$ This patient had bilateral OCA in 2000; both knees were converted to TKA in 2014.

patellectomy (Table 3). The median time to failure was 3.5 years (range $=0.5-13.7$ years), and the mean age of patients with failed OCAs was 42.2 years (range $=17.3-66.0$ years).

Characteristics distinguishing OCA success versus failure included diagnosis, graft location, and graft size. Nine patients of the cohort were treated for degenerative chondral pathology (e.g., degenerative chondral lesion or osteoarthritis) and 6 of these went on to failure $(6 / 9,66.7 \%)$. Five of the 6 failed OCA were isolated to the patellofemoral compartment. Survivorship in this subset of patients with degenerative pathology was $53 \%$ at 5 years and $20 \%$ at 10 years. Median time to failure for these 6 knees was 3.1 years (range $=1.1-9$ years). In addition, mean graft size of failures was larger than that of OCA, which remained in situ at latest follow-up $\left(18.2 \mathrm{~cm}^{2}\right.$ vs. $7.8 \mathrm{~cm}^{2}$, respectively).

\section{Discussion}

The results of this study demonstrate that OCA transplantation is an acceptable primary treatment method for some chondral and osteochondral defects of the knee. Postoperative outcomes scores were improved compared to preoperative scores, and the majority of patients $(84.5 \%)$ were rated as excellent or good at latest follow-up (mean $=7.6$ years). OCA survivorship was high with $89.5 \%$ at 5 years and $74.7 \%$ at 10 years. The incidence of additional surgeries after the initial OCA transplantation was relatively low with 10 patients $(10 / 55,18.2 \%)$ having surgical procedures to address OCA failure and 7 patients $(7 / 55,12.7 \%)$ having surgical procedures performed in conjunction with a functional graft. While the decision to perform an OCA procedure, instead of an alternative surgical cartilage repair treatment, was based on many variables, including size and characteristics of the lesions, OCAs were generally large $\left(\right.$ mean $\left.=9.6 \mathrm{~cm}^{2}\right)$ and had associated subchondral bone involvement. The decision to use OCA transplantation as a "first-line" treatment for these large and complex lesions appears to be supported by the findings of this study.

The use of this case series to study the acceptability of OCA transplantation as a primary treatment option involved the consideration of a number of issues. Although the study was prospective in nature, no control or comparison group was studied and historical controls in the literature are inadequate given that no studies specifically address the use of OCAs as a primary treatment modality. While postoperative $\mathrm{x}$-rays may provide beneficial radiographic follow-up data, clinical outcomes and failure rates were chosen as the postoperative evaluations to provide a more useful metric of the acceptability of OCA transplantation. Given the relatively small sample size in this study, it is impossible to perform a multivariate analysis do determine factors associated with OCA transplantation success or failure. However, there was a trend in failures associated with older patients that had more advanced disease, particularly in the patellofemoral joint.

The survivorship results (i.e., $\sim 90 \%$ at 5 years and $\sim 75 \%$ at 10 years) of the current study are generally consistent with previously reported long-term outcome data of OCA transplantation in the literature; however, the majority of patients described in the literature have had one or more surgical interventions prior to OCA transplantation. OCA survivorship was $\sim 95 \%$ at 5 years, $\sim 80 \%$ to $85 \%$ at 10 years, $\sim 75 \%$ at 15 years, and $\sim 65 \%$ at 20 years in patients with femoral condyle lesions, which was higher than OCA survivorship in patients with patellofemoral lesions ( $\sim 95 \%$ at 5 years, $\sim 70 \%$ to $80 \%$ at 10 years, and $65 \%$ at 15 years). ${ }^{10,11,13}$ In these long-term follow-up studies of OCA transplantation, the majority of knees had one or more surgeries prior to the OCA; 18 patients had 52 operations, ${ }^{11} 122$ patients had 207 operations, ${ }^{13}$ and $14 / 28$ patients had an average of 1.5 previous surgeries. ${ }^{8}$ Historically, over $90 \%$ of patients at our institution undergoing OCA have had a prior surgical intervention. The subset of patients described in this study are 
unique only in the fact that patients did not undergo previous surgical treatment of the condition prior to OCA transplantation. Our previously described success with OCA transplantation used in a variety of conditions encouraged us to utilize OCA transplantation as a first-line treatment. The results reported in this study indicate that success is equivalent to other reported results and that failure of a previous surgical intervention is not a prerequisite for OCA transplantation.

The clinical success demonstrated in the patient population studied here suggests that candidates for primary OCA transplantation are unique compared to other cartilage repair procedures (larger lesions, more bone involvement). While success rates of microfrature, $\mathrm{ACI}$, and OAT can be high, ${ }^{3,4,18-21}$ these interventions are most often use to treat small chondral defects. ${ }^{17}$ The large graft size (mean area $=9.6 \mathrm{~cm}^{2}$ ) and subchondral bone pathology associated with OCD, and AVN, and DJD diagnoses in this study group may better be served by treatment with OCA transplantation compared to other interventions. Lesions that are degenerative in etiology, large in size, or located in the patellofemoral region typically perform worse than those treated for isolated, focal defects as seen here and in the literature..$^{11,15,18}$

\section{Conclusion}

OCA transplantation has historically been considered a salvage procedure for use when other repair treatments have failed. The results of this study suggest that OCA transplantation is a safe and effective primary treatment for many large chondral and osteochondral defects of the knee. Thus, previous surgical intervention is not a prerequisite for treatment of patients with OCA transplantation.

\section{Acknowledgments and Funding}

The authors would like to thank Julie McCauley for help in preparation of this article and Andrea Pallante-Kichura, $\mathrm{PhD}$, for technical writing assistance. The work described in this article was supported by a grant from Scripps Clinic Medical Group.

\section{Ethical Approval}

This research was reviewed and approved by the Institutional Review Board of Scripps Healthcare.

\section{Declaration of Conflicting Interests}

The author(s) declared the following potential conflicts of interest with respect to the research, authorship, and/or publication of this article: Dr. Bugbee's disclosure is Joint Restoration Foundation.

\section{References}

1. Alford JW, Cole BJ. Cartilage restoration, part 2: techniques, outcomes, and future directions. Am J Sports Med. 2005;33:443-60.

2. Mithoefer K, McAdams T, Williams RJ, Kreuz PC, Mandelbaum BR. Clinical efficacy of the microfracture technique for articular cartilage repair in the knee: an evidencebased systematic analysis. Am J Sports Med. 2009;37:2053-63.
3. Steadman JR, Briggs KK, Rodrigo JJ, Kocher MS, Gill TJ, Rodkey WG. Outcomes of microfracture for traumatic chondral defects of the knee: average 11-year follow-up. Arthroscopy. 2003;19:477-84.

4. Gudas R, Gudaite A, Pocius A, Gudiene A, Cekanauskas $\mathrm{E}$, Monastyreckiene E, et al. Ten-year follow-up of a prospective, randomized clinical study of mosaic osteochondral autologous transplantation versus microfracture for the treatment of osteochondral defects in the knee joint of athletes. Am J Sports Med. 2012;40:2499-508.

5. Bugbee WD. Fresh osteochondral allografts. J Knee Surg. 2002;15:191-5.

6. Emmerson BC, Gortz S, Jamali AA, Chung C, Amiel D, Bugbee WD. Fresh osteochondral allografting in the treatment of osteochondritis dissecans of the femoral condyle. Am J Sports Med. 2007;35:907-14.

7. Ghazavi MT, Pritzker KP, Davis AM, Gross AE. Fresh osteochondral allografts for post-traumatic osteochondral defects of the knee. J Bone Joint Surg Br. 1997;79:1008-13.

8. Gortz S, De Young AJ, Bugbee WD. Fresh osteochondral allografting for steroid-associated osteonecrosis of the femoral condyles. Clin Orthop Relat Res. 2010;468:1269-78.

9. Gross AE, Kim W, Las Heras F, Backstein D, SafirO, Pritzker KP. Fresh osteochondral allografts for posttraumatic knee defects: long-term follow-up. Clin Orthop Relat Res. 2008;466:1863-70.

10. Gross AE, Shasha N, Aubin P. Long-term follow-up of the use of fresh osteochondral allografts for posttraumatic knee defects. Clin Orthop Relat Res. 2005;(435):79-87.

11. Jamali AA, Emmerson BC, Chung C, Convery FR, Bugbee WD. Fresh osteochondral allografts: results in the patellofemoral joint. Clin Orthop Relat Res. 2005;(437):176-85.

12. LaPrade RF, Botker J, Herzog M, Agel J. Refrigerated osteoarticular allografts to treat articular cartilage defects of the femoral condyles. A prospective outcomes study. J Bone Joint Surg Am. 2009;91:805-11.

13. Levy YD, Gortz S, Pulido PA, McCauley JC, Bugbee WD. Do fresh osteochondral allografts successfully treat femoral condyle lesions? Clin Orthop Relat Res. 2013;471:231-7.

14. Lyon R, Nissen C, Liu XC, Curtin B. Can fresh osteochondral allografts restore function in juveniles with osteochondritis dissecans of the knee? Clin Orthop Relat Res. 2013;471:1166-73.

15. Torga Spak R, Teitge RA. Fresh osteochondral allografts for patellofemoral arthritis: long-term follow-up. Clin Orthop Relat Res. 2006;444:193-200.

16. Lexer E. Free transplantation. Ann Surg. 1914;60:166-94.

17. Gortz S, Bugbee WD. Allografts in articular cartilage repair. J Bone Joint Surg Am. 2006;88:1374-84.

18. Gobbi A, Kon E, Berruto M, Filardo G, Delcogliano M, Boldrini $\mathrm{L}$, et al. Patellofemoral full-thickness chondral defects treated with second-generation autologous chondrocyte implantation: results at 5 years follow-up. Am J Sports Med. 2009;37:1083-92.

19. Knutsen G, Drogset JO, Engebretsen L, Grontvedt T, Isaksen $\mathrm{V}$, Ludvigsen TC, et al. A randomized trial comparing autologous chondrocyte implantation with microfracture. Findings at five years. J Bone Joint Surg Am. 2007;89:2105-12.

20. Minas T. Autologous chondrocyte implantation for focal chondral defects of the knee. Clin Orthop Relat Res. 2001;(391 suppl):S349-61.

21. McNickle AG, L'Heureux DR, Yanke AB, Cole BJ. Outcomes of autologous chondrocyte implantation in a diverse patient population. Am J Sports Med. 2009;37:1344-50. 